\title{
HIV and breast cancer - a mammographic analysis: An observational study to identify the mammographic pattern of breast cancer in $\mathrm{HIV}$-positive patients
}

\author{
A Vanmali, ${ }^{1} \mathrm{MB}$ ChB, FC Rad Diag (SA); I Buccimazza, ${ }^{2}$ MB ChB, FCS (SA), FACS \\ ${ }^{1}$ Department of Radiology, Nelson R Mandela School of Medicine, College of Health Sciences, University of KwaZulu-Natal, Durban, South Africa \\ ${ }^{2}$ Breast Unit, Department of General Surgery, Nelson R Mandela School of Medicine, College of Health Sciences, University of KwaZulu-Natal, \\ Durban, South Africa
}

Corresponding author: A Vanmali (atishvan@gmail.com)

Background. Data on the association between HIV and breast cancer mammographic patterns and histological subtypes are limited. Objectives. To determine whether specific mammographic findings, histological features and patient profiles were unique to a cohort of HIV-positive patients who developed breast cancer, by comparing them with a HIV-negative cohort.

Methods. This was a descriptive study in which we conducted a retrospective chart review and mammographic and pathology analysis of newly diagnosed breast cancer patients referred to the Addington Hospital breast clinic between August 2008 and June 2012 and entered into a prospective database.

Results. Thirty-eight HIV-positive and 38 HIV-negative patients were included in the study. HIV-positive patients were more likely to have multifocal breast cancer $(p=0.007)$, but not multicentric disease $(p=0.05)$. The presence of grouped and fine pleomorphic microcalcifications and positive HIV status demonstrated statistical significance $(p=0.000)$. A statistically significant relationship between grouped and fine pleomorphic microcalcifications with biopsies confirming high-grade ductal carcinoma in situ (HGDCIS) and HIV status was demonstrated $(p=0.001)$. The mean age of the HIV-positive patients was 42.5 years $(p=0.000)$.

Conclusions. We demonstrated a statistically significant relationship between HIV status, the presence of multifocal breast cancer, and mammographically detected grouped and fine pleomorphic microcalcifications. A statistically significant relationship between HGDCIS and HIV status, and the presence of grouped and fine pleomorphic microcalcifications in HIV-positive patients with biopsies confirming HGDCIS, was demonstrated. Our study also showed that there is a relationship between age of presentation and HIV status.

S Afr Med J 2020;110(2):118-122. https://doi.org/10.7196/SAMJ.2020.v110i2.14149

Breast cancer is the leading cause of cancer-related deaths among females in the developing world. ${ }^{[1]}$ The South African (SA) incidence of breast cancer is $22.2 \%$ of all cancers, with 29.99 adjusted cases per 10000 per year. $^{[2]}$ The most recent statistics, from 2012, demonstrated that 9815 women were diagnosed with breast cancer, and 3848 died from the disease. ${ }^{[3]}$ Breast cancer is the most prevalent type of nonAIDS-defining cancer in HIV-positive women. ${ }^{[4]}$

The total number of persons living with HIV in SA increased from an estimated 4.25 million in 2002 to 7.52 million by 2018. In 2018, an estimated $13.1 \%$ of the total population, and approximately one-fifth of women of reproductive age (15 - 49 years), were HIV-positive. ${ }^{[5]}$ According to UNAIDS (Joint United Nations Programme on HIV/ AIDS) data for 2017, 51\% of people living with HIV/AIDS globally were female. ${ }^{[6]}$ The 2017 South African National HIV Prevalence, Incidence and Behaviour Survey ${ }^{[7]}$ revealed that, based on point estimates, the overall HIV peak prevalence was $31.5 \%$ in 35 - 39-yearolds, but differed by gender (females at $39.4 \%$ and males at $23.7 \%$ ) and peaked at an older age among males (45 - 49 years) compared with females ( 35 - 39 years).

HIV-infected individuals are at an increased risk of developing non-AIDS-defining cancers. ${ }^{[8]}$ Despite extensive research in the field of HIV, data on breast cancer incidence, stage at presentation, response to treatment, prognosis, survival, mammographic patterns, histological subtypes and the role of screening in this patient population are limited.
In a study of breast cancer characteristics and HIV in 1092 women in Soweto, SA, one-third of patients aged $<50$ years were HIVpositive. These findings highlight the need for HIV testing of breast cancer patients, especially young patients, in HIV-endemic populations. ${ }^{[9]}$

In a study between the years 1998 and 2007 by Pantanowitz et al. ${ }^{[10]}$ the median age of HIV-positive patients with breast cancer was 45 years, and most carcinomas were high-grade cancers.

In the present study, we hypothesised that specific mammographic features develop early in the pathogenesis of breast cancer in HIVpositive women. We further postulated that in HIV-positive women who develop breast cancer, the process initially begins as a high-grade ductal carcinoma in situ (HGDCIS). These HIV-positive women are not generally included in screening programmes owing to their young age, so the occult pre-invasive disease is not detected and progresses to an invasive carcinoma. Our hypothesis is that HIV may be associated with HGDCIS, which is known to proceed to invasive breast cancer within 5 years if untreated.

\section{Objectives}

The primary objective of this study was to identify specific mammographic patterns and features in a cohort of HIV-positive patients with breast cancer and compare these features with an HIV-negative cohort, to determine whether the presence of certain mammographic features was unique to one or other of the cohorts. 
Secondary objectives included establishing whether there was a difference in the average age of presentation of the two groups and determining the spectrum of histology (invasive ductal or lobular carcinoma) of breast cancer in HIV-positive patients. We further investigated whether a particular histological subtype predominates, particularly where an associated HGDCIS is suspected based on certain radiological features.

\section{Methods}

This was a descriptive study in which a retrospective chart review of all newly diagnosed breast cancer patients referred to the Addington Hospital breast clinic between August 2008 and June 2012 and entered into a prospective database was conducted.

All patients with histologically proven breast cancer and a mammogram who were either known to be HIV-positive or found to be HIV-positive on subsequent testing were included in the study. A randomly selected group of HIV-negative patients formed the comparison group. All pathology specimens that could be traced to the respective patients (28 HIV-positive patients and $31 \mathrm{HIV}$-negative patients) were used for analysis. Male patients and HIV-positive patients without a mammogram (not performed owing to technical reasons, or missing at the time of analysis) were excluded.

Mammograms were subjected to double reading by two specialist radiologists with an interest in breast imaging and blinded to identifying patient features, in accordance with international guidelines (American College of Radiology Breast Imaging Reporting and Data System (BI-RADS), 2003 (4th edition) ${ }^{[11]}$ ). Since this study was conducted in 2012, and preceded the BI-RADS 5th edition, we used the 2003 descriptive lexicon. A list of patient numbers was submitted to the designated anatomical pathologist, who independently reviewed the specimens and subsequently forwarded the histology results. This process reduced inter-observer variability of histology reports, which were previously reviewed by random pathologists. Breast cancers were defined as multifocal when more than one distinct tumour was detected in the same quadrant and as multicentric when multiple distinct tumours were present in different quadrants of the breast.

The ages of the patients were captured to analyse the average age at presentation. Data extracted from the double reading of the mammogram included characterisation of any mass lesion if present, the presence of variable patterns of malignant microcalcification (grouped, fine pleomorphic, coarse heterogeneous, fine linear and fine branching), the presence of multifocal and multicentric lesions, and the presence of lesions involving both breasts. All lesions, whether clinically palpable or occult, were biopsied under image guidance. This corresponded to the mammographically detected lesion. Owing to the retrospective nature of the study, it was not possible to determine whether all multifocal and multicentric lesions were biopsied. Histological analysis of the biopsy specimen from the mammographically documented lesion was performed, and the presence or absence of HGDCIS was noted. A correlation of histologically proven HGDCIS and the corresponding features on the mammogram was performed to elucidate whether the presence of malignant microcalcification might suggest the presence of HGDCIS.

Anonymity was maintained by the use of study numbers instead of patient identifiers. Each patient was allocated a study number. The data sheet that linked each patient to the study number was secured in the principal investigator (PI)'s offices, and access was limited to the PI (AV) and co-investigator. The computerised version was kept secure by password-protected access. Knowledge of patient identity was limited to the PI and co-investigator.
Institutional approval was obtained from the Addington Hospital and Inkosi Albert Luthuli Central Hospital management and the University of KwaZulu-Natal Biomedical Research Ethics Committee (ref. no. BE013/13).

\section{Statistical analysis}

The $\chi^{2}$ test $(p<0.05)$ was used to assess the statistical relationship between the two categorical variables of HIV status and mammographic features. These features included the distribution of breast cancer (multifocal and multicentric disease) and presence of the malignant patterns of microcalcifications. The two-sample $t$-test was used to determine the difference in the average age of presentation of HIVpositive and HIV-negative patients. Fisher's exact test with a 95\% confidence interval was applied to assess the statistical significance of the presence or absence of bilateral breast cancer.

\section{Results}

The initial group contained 90 HIV-positive patients; however, during data collection many of the mammograms were not available, and 52 patients were therefore excluded from the study. The study group comprised $38 \mathrm{HIV}$-positive patients, and the comparison group consisted of 38 randomly selected HIV-negative patients. Only 28 HIV-positive and 31 HIV-negative pathology specimens were available in the respective arms of the study.

Using the two-sample $t$-test, we accepted that there was a difference in the average age of presentation of HIV-positive and HIV-negative patients, with HIV-positive patients being significantly younger than those who were HIV-negative $(p=0.000)$ (Table 1 ).

Ten patients had mammographic features suggestive of multifocal breast cancer. Of these, 9 were HIV-positive. A statistically significant relationship between HIV status and multifocal disease was demonstrated on $\chi^{2}$ analysis $(p=0.007)$ (Table 2).

A total of 17 patients presented with mammographic features of multicentric breast cancer, and of these 12 were HIV-positive. In the assessment of multicentric disease the $p$-value was 0.05 , which is not statistically significant (Table 3).

Of the 28 patients with fine pleomorphic microcalcification and the 26 with grouped microcalcification, 22 were HIV-positive in each group. In analysing the patterns of microcalcification, our data demonstrated statistical significance between grouped microcalcification $(p=0.000)$ and fine pleomorphic microcalcification $(p=0.000)$ and HIV status (Tables 4 and 5 ).

Twenty-eight pathology specimens from the HIV-positive group and 31 from the HIV-negative group were obtained for analysis by the independent pathologist. When the presence of HGDCIS was correlated with HIV status, statistical significance between the presence of HGDCIS and HIV status was demonstrated $(p=0.012)$ (Table 6).

Using the same analysis, our data demonstrated a statistically significant relationship between the presence of grouped and fine pleomorphic microcalcifications with biopsies confirming HGDCIS and HIV status ( $p=0.001)$ (Table 7).

We failed to demonstrate statistical significance in the relationship between bilateral breast cancer and HIV status ( $p=0.753$ ) (Table 8).

\section{Table 1. Relationship between mean age and HIV status}

\begin{tabular}{lll}
\hline HIV status & Patients, $\boldsymbol{n}$ & Age (years), mean \\
\hline Positive & 38 & 42.5 \\
Negative & 38 & 57.6 \\
Total & 76 & 50.1
\end{tabular}


Table 2. Relationship between the presence of mammographic features of multifocal breast cancer and HIV status

\begin{tabular}{llll}
\hline HIV status & Multifocal disease, $\boldsymbol{n}(\%)$ & No multifocal disease, $\boldsymbol{n}(\%)$ & Total, $\boldsymbol{N}$ \\
\hline Positive & $9(90.0)$ & $29(43.9)$ & 38 \\
Negative & $1(10.0)$ & $37(56.1)$ & 38 \\
Total & $10(100)$ & $66(100)$ & 76
\end{tabular}

Table 3. Relationship between the presence of mammographic features of multicentric breast cancer and HIV status

\begin{tabular}{llll}
\hline HIV status & Multicentric disease, $\boldsymbol{n}(\%)$ & No multicentric disease, $\boldsymbol{n}(\%)$ & Total, $\boldsymbol{N}$ \\
\hline Positive & $12(70.6)$ & $26(44.1)$ & 38 \\
Negative & $5(29.4)$ & $33(55.9)$ & 38 \\
Total & $17(100)$ & $59(100)$ & 76
\end{tabular}

Table 4. Relationship between the presence of fine pleomorphic microcalcification and HIV status

\begin{tabular}{llll}
\hline & \multicolumn{2}{l}{ Fine pleomorphic microcalcification, } & \multicolumn{2}{l}{ No fine pleomorphic microcalcification, } \\
HIV status & $\boldsymbol{n}(\%)$ & $16(33.3)$ & Total, $\boldsymbol{N}$ \\
\hline Positive & $22(78.6)$ & $32(66.7)$ & 38 \\
Negative & $6(21.4)$ & $48(100)$ & 38 \\
Total & $28(100)$ & & 76
\end{tabular}

Table 5. Relationship between the presence of grouped microcalcification and HIV status

\begin{tabular}{llll}
\hline HIV status & Grouped microcalcification, $\boldsymbol{n}(\%)$ & No grouped microcalcification, $\boldsymbol{n}$ (\%) & Total, $\boldsymbol{N}$ \\
\hline Positive & $22(84.6)$ & $16(32.0)$ & 38 \\
Negative & $4(15.4)$ & $34(68.0)$ & 38 \\
Total & $26(100)$ & $50(100)$ & 76
\end{tabular}

Table 6. Prevalence of HGDCIS in HIV-positive and HIV-negative patients

\begin{tabular}{llll}
\hline HGDCIS & HIV-negative, $\boldsymbol{n}(\%)$ & HIV-positive, $\boldsymbol{n}(\%)$ & Total, $\boldsymbol{N}$ \\
\hline Absent & $19(61.3)$ & $8(28.6)$ & 27 \\
Present & $12(38.7)$ & $20(71.4)$ & 32 \\
Total & $31(100)$ & $28(100)$ & 59 \\
HGDCIS = high-grade ductal carcinoma $i n$ situ. & &
\end{tabular}

Table 7. Prevalence of grouped and fine pleomorphic microcalcifications with HGDCIS

\begin{tabular}{llll}
\hline Grouped and fine pleomorphic & & HIV-positive, $\boldsymbol{n}$ (\%) & Total, $\boldsymbol{N}$ \\
\hline microcalcifications with HGDCIS & HIV-negative, $\boldsymbol{n}$ (\%) & $5(25.0)$ & 15 \\
Absent & $10(83.3)$ & $15(75.0)$ & 17 \\
Present & $2(11.7)$ & $20(100)$ & 32 \\
Total & $12(100)$ & & \\
HGDCIS = high-grade ductal carcinoma in situ. & &
\end{tabular}

Table 8. Relationship between breast cancer and laterality

\begin{tabular}{llll}
\hline HIV status & Both breasts, $\boldsymbol{n}(\%)$ & Single breast, $\boldsymbol{n}(\%)$ & Total, $\boldsymbol{N}$ \\
\hline Positive & $1(50.0)$ & $37(50.0)$ & 38 \\
Negative & $1(50.0)$ & $37(50.0)$ & 38 \\
Total & $2(100)$ & $74(100)$ & 76
\end{tabular}

\section{Discussion}

A literature review failed to reveal any study that analysed the presence or absence of specific mammographic patterns in HIVpositive patients with breast cancer. However, clinical practice and multidisciplinary meetings at our institution detected a trend in mammographic patterns among HIV-positive patients with breast cancer, prompting a retrospective analysis of the mammograms and pathology reports of these patients compared with an HIV-negative cohort. Our findings suggest that HIV-positive and HIV-negative patients present with different mammographic and histological profiles.

The primary objective of our study was to identify specific mammographic patterns and features in HIV-positive and HIVnegative patients with breast cancer and to determine whether the presence of certain mammographic features was unique to one or other of the cohorts. 
Our data demonstrated a statistically significant relationship between multifocal breast cancer and HIV status. HIV-positive patients were more likely to present with multifocal breast cancer compared with HIV-negative patients. This relationship was statistically significant $(p=0.007)$.

The presence of multicentric breast cancer and breast cancer affecting both breasts in HIV-positive and HIV-negative patients failed to reach statistical significance. However, we continue to see a large number of multicentric breast cancers and bilateral breast cancers in our clinical practice. A prospective study with a more extensive study group and detailed record-keeping is warranted to investigate this association further.

The importance of distribution of disease was well demonstrated by Neri et al. ${ }^{[12]}$ who demonstrated that multifocal and multicentric disease were biologically more aggressive than unifocal tumours, had an increased propensity to metastatic diffusion, and were associated with worse outcomes. The HIV status of the patients was not considered as a parameter in their study. Ki-67 antigen is a marker of cell proliferation and is currently used as a prognostic marker in the management of breast cancer. Tumour biology (molecular subtype and Ki-67 antigen) was not captured in our study, and follow-up was not an end-point. It was therefore not possible for us to relate HIV status to more aggressive disease or a worse outcome.

With regard to patterns of microcalcification and HIV status, we demonstrated a statistically significant correlation between the presence of fine pleomorphic microcalcification $(p=0.000)$ and grouped microcalcification $(p=0.000)$ in the HIV-positive group.

The findings of grouped and fine pleomorphic microcalcifications were incorporated in the pathology analysis to determine whether these mammographic findings demonstrated any correlation with the pathology results and known HIV status. Our data demonstrated a statistically significant relationship between the presence of grouped and fine pleomorphic microcalcifications with biopsies confirming HGDCIS in HIV-positive patients ( $p=0.001$ ).

Our study also demonstrated a relationship between HGDCIS and HIV status. Ductal carcinoma in situ (DCIS) is a heterogenous disease and a non-obligate precursor to invasive carcinoma. Currently there is controversy over the management of intermediate- and low-grade DCIS, with some researchers believing that it will probably never cause clinical disease; however, HGDCIS has been known to progress to invasive ductal carcinoma within 5 years if untreated. We are uncertain whether there is a link between HIV and the genesis of HGDCIS, but in our study, HIV-positive patients were more likely to harbour associated HGDCIS than HIV-negative patients $(p=0.012)$, supporting our hypothesis of the predominance of a particular pre-invasive histological type (HGDCIS) in HIV-positive patients. These findings strengthen our hypothesis suggesting a link, but this postulate needs to be subjected to a larger prospective study.

The presence of mammographic features such as grouped and fine pleomorphic microcalcifications and the predominance of HGDCIS in our subset of HIV-positive patients warrant further investigation to determine whether HGDCIS is an obligate precursor to invasive duct carcinoma in HIV-positive patients.

The apparent clinical trend of HIV-positive patients presenting with breast cancer at a younger age than HIV-negative patients was analysed as a secondary objective. Our data demonstrated a mean age of 42.5 years in the HIV-positive group and 57.6 years in the HIV-negative group. Using appropriate statistical analysis, we accepted that there is a difference in the average age of presentation of HIV-positive and HIV-negative patients, with HIV-positive patients being significantly younger than those who were HIVnegative $(p=0.000)$.
Two case reports have suggested that HIV-associated breast cancer demonstrated atypical characteristics of earlier age of onset (36 and 35 years of age, respectively) and marked aggressiveness of the cancer. ${ }^{[13]}$ Voutsadakis and Silverman ${ }^{[14]}$ had similar findings, reporting four cases demonstrating that breast cancer was more aggressive in HIV-positive patients than in those who were HIVnegative. The present study did not include the characteristics of breast cancer, but we did assess the mean age of onset of breast cancer in HIV-positive and negative patients, as described above.

In one of the larger series documented to date, Hurley et al. ${ }^{[15]}$ documented $20 \mathrm{HIV}$-positive patients with breast cancer between the years 1988 and 2001. In contrast to previous reports, these patients were older, with an average age of 44 years, and the distribution in terms of stage was similar to the general population.

In a study between the years 1998 and 2007, the median age at which breast cancer was diagnosed in HIV-positive patients was 45 years, and most carcinomas were of high grade. ${ }^{[10]}$ In the ONCOVIH study of breast cancer among HIV-infected individuals, the median age was 43.8 years. ${ }^{[16]}$ In an SA study of 1092 women with breast cancer, one-third of patients aged $<50$ years were HIV-positive. These findings highlight the need for HIV testing of breast cancer patients, especially young patients, in HIV-endemic populations. ${ }^{[9]}$ The most striking finding of a recent study at the Dr George Mukhari Academic Hospital breast clinic in Pretoria, SA, ${ }^{[17]}$ concerns age at presentation with breast cancer, which varied according to HIV status: the mean (standard deviation) age at presentation in the HIVpositive group ( $44.86(9.00)$ years) was $\sim 10$ years younger than that in the HIV-negative group (53.18 (12.48) years) and that of the general population $(54.56(13.62)$ years) $(p<0.0001)$. This finding raises the question whether HIV-positive patients should be screened for breast cancer at a younger age than the general population. ${ }^{[17]}$

\section{Study limitations}

The retrospective nature of our study and the small numbers in each group are significant limitations of our study.

Lack of adequate record-keeping of radiological studies and untraceable pathology specimens excluded a large number of patients from the study and resulted in the inability to link pathology results to $10 \mathrm{HIV}$-positive (20.8\%) and $7 \mathrm{HIV}$-negative patients (15.5\%) with available mammograms.

Histological correlation was obtained from available pathology specimens. We were unable to link every mammographically detected lesion with a pathology specimen, and consequently the descriptions of multifocal and multicentric lesions demonstrating malignant features on the mammogram were not all supported by histological confirmation.

An age-matched cohort analysis of the two groups was not performed.

Multivariate analysis was not performed to establish whether the mammographic features and the HGDCIS diagnosis were independent of age.

These limitations will be interrogated in the follow-up prospective study.

\section{Conclusions}

Our study demonstrated a statistically significant relationship between HIV status and the presence of mammographically detected multifocal breast cancer, and grouped and fine pleomorphic microcalcifications, in HIV-positive patients. We further demonstrated a statistically significant relationship between the presence of HGDCIS and HIV status, as well as the presence of HGDCIS in grouped and fine pleomorphic microcalcifications in HIV-positive patients. 
A prospective age-matched study with a significantly larger study population is required to explore the possible relationship between grouped and fine pleomorphic microcalcifications and high-grade DCIS in HIV-positive patients diagnosed with invasive breast cancer. An association between HGDCIS in suspicious microcalcifications and HIV-positive patients diagnosed with invasive breast cancer may have implications for screening programmes and earlier breast cancer detection in this high-risk population. Currently there is no national screening programme in SA. However, our hypothesis that HIV-positive patients are prone to HGDCIS requires interrogation. If a larger prospective study identifies a link between HIV-positive status and HGDCIS, guidelines to commence earlier radiographic screening can be designed in this cohort of patients.

Declaration. Acceptance of this article for publication served to fulfil a requirement for AV's MMed (Radiology) degree.

Acknowledgements. We extend our gratitude to Dr L Y Perumal for his significant assistance in the double reading of the mammograms. We also thank Prof. P K Ramdial for her detailed analysis of all pathology specimens, allowing us to improve the objectivity of the study

Author contributions. AV made substantial contributions to the conception and design of the study. He wrote the research proposal, acquired, analysed and interpreted the data, drafted the manuscript and approved the final version to be published. IB made a substantial contribution to the conception and design of the study, assisted in drafting of the research proposal and in the analysis and interpretation of the data, critically revised the manuscript for important intellectual content, and approved the final version to be published.

Funding. None.

Conflicts of interest. None.
1. Torre L, Bray F, Siegel R, Ferlay J, Lortet-Tieulent J, Jemal A. Global cancer statistics, 2012. CA Cancer J Clin 2015;65(2):87-108. https://doi.org/10.3322/caac.21262

2. National Cancer Registry, South Africa. 2013. http://www.nicd.ac.za/wp-content/uploads/ 2017/03/2013NCR.pdf (accessed 22 February 2019).

3. Gray A, Vawda Y. Health policy and legislation. In: Padarath A, Barron P, eds. South African Health Gray A, Vawda Y. Health policy and legislation. In: Padarath A, Barron P, eds. South African Health
Review 2017. Durban: Health Systems Trust, 2017. http://www.hst.org.za/publications/south-africanReview 2017. Durban: Health Systems Trust, 2017.

4. Grover S, Martei Y, Puri P, et al. Breast cancer and HIV in sub-Saharan Africa: A complex relationship. Grover S, Martei Y, Puri P, et al. Breast cancer and HIV in sub-Saharan
J Glob Oncol 2018; (4):1-11. https://doi.org/10.1200/JGO.2016.006585

J Glob Oncol 2018;(4):1-11. https://doi.org/10.1200/JGO.2016.006585
Statistics South Africa. Mid-year population estimates 2018. Statistical release P0302. Pretoria: Stats Statistics South Africa. Mid-year population estimates 2018. Statistical release P0302. Pretoria: Stats
SA, 2019. http://www.statssa.gov.za/publications/P0302/P03022018.pdf (accessed 22 February 2019). SA, 2019. http://www.statssa.gov.za/publications/P0302/P03022018.pdf (accessed 22 February 2019). 6. Joint United Nations Programme on HIV/AIDS (UNAIDS). UNAIDS Data 2017. https://www.unaid
org/sites/default/files/media_asset/20170720_Data_book_2017_en.pdf (accessed 22 February 2019). 7. The Fifth South African National HIV Prevalence, Incidence, Behaviour and Communication Survey, 2017 (SABSSM V). Aidshealth.org 2018. https://www.aidshealth.org/wp-content/uploads/2018/08/ hsrc-survey-2018-summary.pdf (accessed 22 February 2019).

8. Latif N, Rana F, Guthrie T. Breast cancer and HIV in the era of highly active antiretroviral therapy: Two case reports and review of the literature. Breast J 2010;17(1):87-92. https://doi.org/10.1111/j.15244741.2010.01023.x

9. Cubasch H, Joffe M, Hanisch R, et al. Breast cancer characteristics and HIV among 1,092 women in Soweto, South Africa. Breast Cancer Res Treat 2013;140(1):177-186. https://doi.org/10.1007/s10549013-2606-y

10. Pantanowitz L, Sen S, Crisi G, Makari-Judson G, Garb J, Skiest D. Spectrum of breast disease encountered in HIV-positive patients at a community teaching hospital. Breast 2011;20(4):303-308 https://doi.org/10.1016/j.breast.2010.08.003

11. American College of Radiology. Breast Imaging Reporting and Data System (BI-RADS). 4th ed. Reston, Va: ACR, 2003

12. Neri A, Marrelli D, Megha T, et al. Clinical significance of multifocal and multicentric breast cancers and choice of surgical treatment: A retrospective study on a series of 1158 cases. BMC Surg 2015:15:1. https://doi.org/10.1186/1471-2482-15-1

13. Cuvier C, Espie M, Extra J, Marty M. Breast cancer and HIV infection: Two case reports. Eur J Cancer 1997;33(3):507-508. https://doi.org/10.1016/S0959-8049(97)89033-8

14. Voutsadakis I, Silverman L. Breast cancer in HIV-positive women: A report of four cases and review of the literature. Cancer Invest 2002;20(4):452-457. https://doi.org/10.1081/CNV-120002144

15. Hurley J, Franco S, Gomez-Fernandez C, et al. Breast cancer and human immunodeficiency virus: 5. Hurley J, Franco S, Gomez-Fernandez C, et al. Breast cancer and human immunodeficiency virus:
A report of 20 cases. Clin Breast Cancer 2001;2(3):215-220. https://doi.org/10.3816/CBC.2001.n.024 16. Spano J, Lanoy E, Mounier N, Katlama C, Costagliola D, Heard I. Breast cancer among HIV
16. Spano J, Lanoy E, Mounier N, Katlama C, Costagliola D, Heard I. Breast cancer among HIV
infected individuals from the ONCOVIH study, in France: Therapeutic implications. Eur J Cancer 2012;48(18):3335-3341. https://doi.org/10.1016/j.ejca.2012.05.019

17. Van Zyl N, Minné C, Mokone D. Human immunodeficiency virus infection in breast cancer patients: The prevalence thereof and its effect on breast cancer characteristics at Dr George Mukhari Academic
Thention in breast cancer patients: The prevalence thereof and its effect on breast cancer characteristics at Dr George Mukhari Academic
Hospital Breast Clinic, Ga-Rankuwa, South Africa. S Afr J Rad 2018;22(2):a1361. https://doi. org/10.4102/sajr.v22i2.1361

Accepted 10 July 2019 